\title{
A Design and Construction of a Wind Tunnel for Engineering Laboratories
}

\author{
Chalida U-tapao ${ }^{1}$ and Seksun Moryadee ${ }^{2}$ \\ ${ }^{1}$ Department of Civil Engineering, Faculty of Engineering, King Mongkut's Institute of Technology Ladkrabang, Bangkok, Thailand \\ ${ }^{2}$ Department of Ordnance Engineering, Chulachomklao Royal Military Academy Nakhon Nayok, Thailand
}

\begin{abstract}
The propose of this research is to demonstrate a design and construction of a wind tunnel for engineering laboratories in order to study the principles and control wind speeds in the wind tunnel. In an experiment in aerodynamics and engineering, we found that diffuser must have a length equal to or more than twice the length of the test in order to prevent the turbulent flow in the test area. The wind speed control system uses Inverter to control a 3-phase frequency of electricity supplied to air blower. In the experiment, the frequency was adjusted in the range from 20.00 to $50.00 \mathrm{~Hz}$. Experiment results show that wind speeds during the test area are in the range of 14.50 to 38.50 meters per second, and the relationship between frequency $(\mathrm{Y})$ and wind speed $(\mathrm{X})$ during the test is linear as follows: $\mathrm{Y}=(0.7945 \times(\mathrm{X}-20))+$ 14.629. The maximum pressure is 90.31 kilograms per square meter. This wind tunnel can be used to design buildings with a maximum height of 20 meters according to the Bangkok Metropolis Building Control (2001).
\end{abstract}

\section{Introduction}

The damage in building structure mainly occurres at the lateral side. The design of equipment in engineering for the building structure in the civil engineering and aerospace engineering must have the standard and rule. The design of building structure for supporting the lateral force is related to the wind force. In Thailand, the Engineering Institute of Thailand (EIT) is an organization in charge of standard and rule of the building structure. Moreover, EIT identifies the standard of the building structure for supporting the lateral force related to the wind force. Therefore, one of the main tasks for EIT is to conduct a research on the lateral force. In order to do that, the realistic generator of the lateral force is design based on small scale for testing the material, equipment and structure. The strength per the lateral force is very important to use in the all engineering.

This research focuses on design and construction of the realistic generator of the lateral force, called the wind tunnel, which can conduct a measurement in wind speed material test and other tests in the laboratory.

\section{Literature Review}

Wilson et, al, (2008) conducted a study on the wind tunnel for testing the sensor in pressures force and wind speeds. The wind tunnel that is uniform laminar flow in the test section. This work applied the test section at diameter of $180 \mathrm{~mm}$ and $120 \mathrm{mmd}$ with height (up to 60 meters per second). The model considers the flow characteristics at the flow entrance of the air by using Reynolds numbers. The result shows that the wind speeds is $3 \%$ within $\pm 0.3 \%$. accuracy. These values are suitable for designing the wind tunnel.

Wittwer and Moller (2000) evaluated the wind tunnel which is low speed of National University of the Northeast (UNNE). This work analyzed the velocity flow and turbulence flow. The output of that research demonstates that velocity is constant and turbulence is low.

Huang et al., (2010) presented the Fuzzified Eigensystem Realization Algorithm (FERA) method. It is used to perform estimation in the set of aerodynamic parameters. The Initial of design fundamental and the result of design detail of Mini - UAV are based on LSWT which is a new method with combined theory fuzzy logic and Eigensystem Realization Algorithm (ERA). There are characteristics of aerodynamic parameters. For the FERA method obtains input variables which is better the testing tunnel realistic.

Boyle (2002) proposed the wind tunnel under uncertainty of data analysis. In the measuremed data an output is a dimensionless. It is an important of objective in design In case of laminar uniform for flowing into the test section impacts the factor. Moreover, the construction of the air flow is turbulence uniform. As the results, the factors affects wind velocity in a construct

\footnotetext{
* Corresponding author: kuchalid@kmitl.ac.th
} 
ion ofwind tunnels with a cross-sections test of the wind tunnel.

\section{Methodology}

This paper proposed a design and construct the wind tunnel. In the first stage the wind tunnel is designed elements. Next, the wind tunnel is constructed with acrylic material. Then, the wind speed value is measured for testing the performance of the wind tunnel according to theory of the wind tunnel.

\subsection{Design Elements}

\subsubsection{Contraction}

The design contraction assigns the length of the contraction between 1 to 1.5 times of the diameter of the entrance as flows:

$$
D_{h} \leq L_{c} \leq 1.5 D_{h}
$$

where

$D_{h}$ is the diameter of hydraulic.

$L_{c}$ is the length of contraction.

\subsubsection{Contraction Ratio}

Contraction Ratio $(C)$ is the ratio of the cross-sectional area which is the entrance per the cross-sectional area of the exit. $C$ is defined between 6 to 9 .

$$
6 \leq C \leq 9
$$

\subsubsection{Test section}

The test section is a ratio of height per the crosssectional area that is in a range of 0.7 to 1 as follows

$$
0.7 \leq \frac{H}{W} \leq 1
$$

where

$H$ is the height of test section.

$W$ is the width of test section.

Equation 4 is the length of the truncation.

$$
L_{T} \geq 2 D_{h}
$$

where

$L_{T}$ is the length of test section

$D_{h}$ is the diameter of hydraulic

Fig. 1 shows the diffuser pile. The ratio of section area $\left(A_{R}\right)$ is defined by

$$
A_{R}=\frac{A_{2}}{A_{1}}
$$

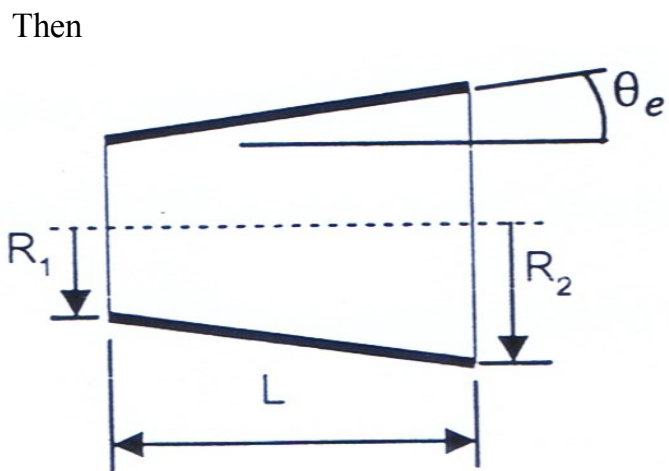

Fig 1. Diffuser pile.

$$
2 \leq A_{R} \leq 3
$$

where

$A_{2}$ is the section area entrance of wind.

$A_{1}$ is the section area exists of wind.

\subsubsection{Slope Angle}

Generally, a slope angle $\theta_{e}$ is the range, we get

$$
\theta_{e}=\tan ^{-1}\left(\frac{R_{2}-R_{1}}{L}\right)=\tan ^{-1}\left(\frac{1}{2} \frac{\sqrt{A_{R}^{-1}}}{L / D_{1}}\right)
$$

then $2^{\circ} \leq \theta_{e} \leq 3.5^{\circ}$.

\subsubsection{Design the Grille}

The width and the shape of the grille affected the reduction of the turbulent flow. Thus, we must select suitable for the wind tunnel.

\subsection{Construction}

In this sub-section presents the construction of the wind tunnel composed of acrylic material, the air duct, test section, diffuser, metal parts, the grate, and fan components as demonstrated in Fig.2. And Fig. 3 shows the completion of the wind tunnel used to test the material.

\subsection{Testing to start a wind tunnel}

\subsubsection{Design and Construction}

The open loop wind tunnel uses inverter to control a 3 phase electricity supply. The performance of this open 
loop wind tunnel is measured for confirming competency before applying to any test. Hence, the testing a wind tunnel is started as follows:

1. Measuring the section test of the wind speed values. The adjusted frequency starts with $20 \mathrm{~Hz}$ increment by 2 Hzs to measure the wind speed values and record the results.

2. Repeat step 1. Bring the wind speed values at the same frequency estimate the average values.

3. We obtain the wind speed values in each frequency as the section test.

4. Plot graph the relationship between the wind speed values each as section test and the frequency.

5. The wind speed values of the section test are collected for calculating the wind force pressure unit.
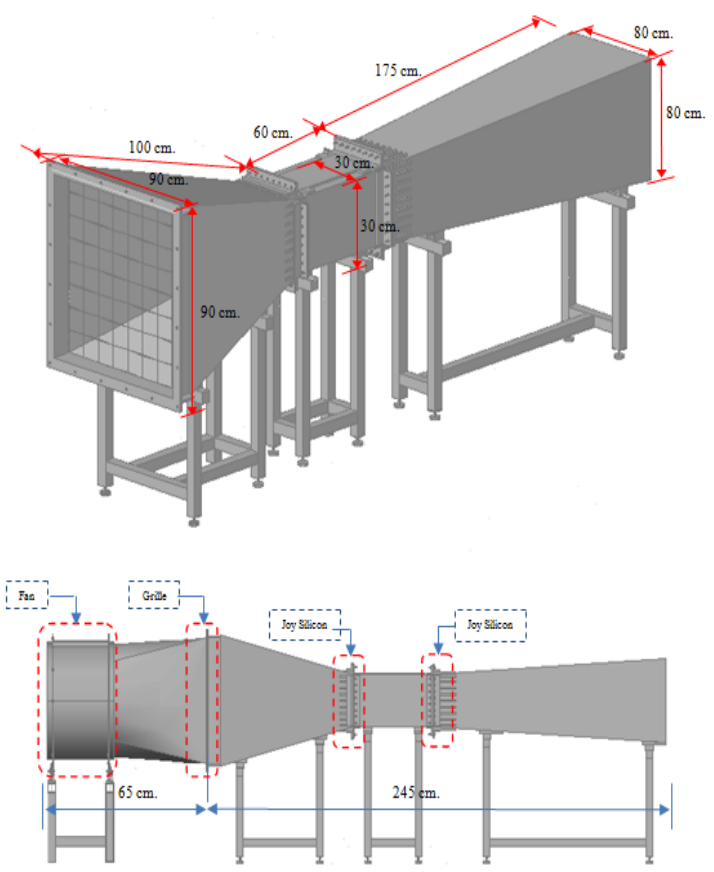

Fig 2. The dimension of the wind tunnel

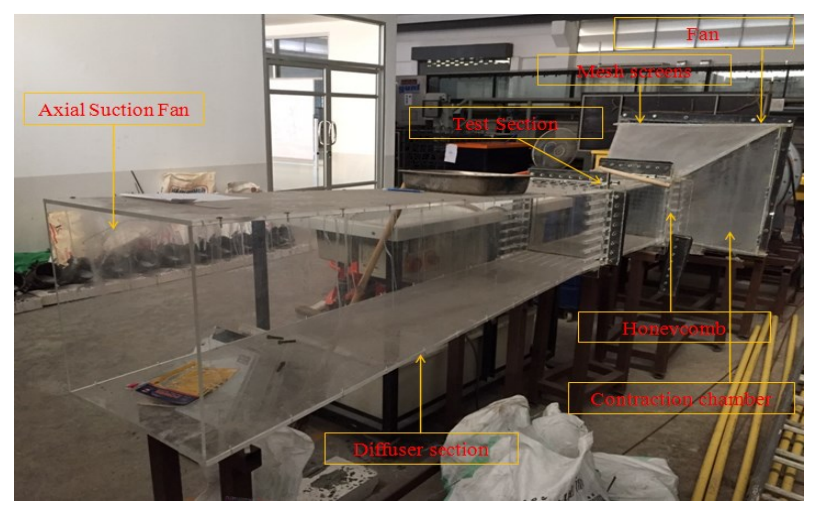

Fig 3. The wind tunnel used to test the material.

\subsubsection{Use of the wind tunnel}

1. The open box test section is used to feed the material test. The materials can be placed at the center of the test section.
2. The material test must not exceed $30 \times 30 \mathrm{~cm}$ for testing the lateral force of the wind tunnel as shown in Fig. 4.

3. Turn on the machine and the inverter fan. Adjusting the frequency between $20 \mathrm{~Hz}$ to $50 \mathrm{~Hz}$ to measure the wind speed of the material test.

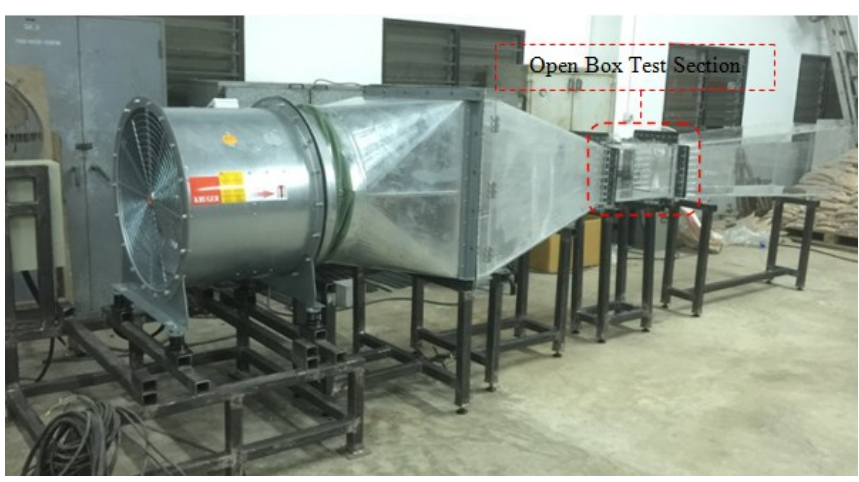

Fig 4. The opened box test section for feeding material.

\section{Results}

In this framework, we present the results of the wind tunnel which can be utilized for using the material testing. This wind tunnel is designed and constructed. The experimental can be formed at the center of the test section, then the performance of the wind tunnel is measured as illustrated in Table 1.

Table1. The experimental results of the wind speed values.

\begin{tabular}{|c|c|c|c|}
\hline \multirow{2}{*}{$\begin{array}{c}\text { Power } \\
\text { supply } \\
\text { (Hzs) }\end{array}$} & \multicolumn{3}{|c|}{ Wind Speed (m/s) } \\
\cline { 2 - 4 } & 1 st time & $\begin{array}{c}\text { 2nd } \\
\text { time }\end{array}$ & Average \\
\hline 20 & 14.50 & 14.7 & 14.60 \\
\hline 22 & 16.1 & 15.9 & 16.00 \\
\hline 24 & 17.5 & 17.8 & 17.65 \\
\hline 26 & 19.2 & 19 & 19.10 \\
\hline 28 & 20.7 & 20.9 & 20.80 \\
\hline 30 & 22.7 & 22.6 & 22.65 \\
\hline 32 & 24.3 & 24.5 & 24.40 \\
\hline 34 & 26.1 & 26.3 & 26.20 \\
\hline 36 & 27.5 & 27.8 & 27.65 \\
\hline 38 & 29.4 & 29.1 & 29.25 \\
\hline 40 & 30.9 & 30.7 & 30.80 \\
\hline 42 & 32.3 & 32.2 & 32.25 \\
\hline 44 & 33.4 & 33.8 & 33.60 \\
\hline 46 & 35.3 & 35.1 & 35.20 \\
\hline 48 & 36.4 & 36.7 & 36.55 \\
\hline 50 & 38.2 & 37.9 & 38.05 \\
\hline
\end{tabular}

Table 1. shows the experiment results of the wind speed values. It can be concluded that this wind tunnel is the testing of wind speed during the test area at frequency in the range 20 to $50 \mathrm{~Hz}$. Next, the results in Table 1. are 
plotted the relationship between the wind speed values and frequency as shown in Fig. 5.

From Fig. 5, the relationship between wind speed values $(\mathrm{Y})$ and frequency $(\mathrm{X})$ is linear as follws:

$$
Y=(0.7945 \times(X-20))+14.629
$$

where

$Y$ is the wind speed of the test section.

$X$ is the electrical frequency power.

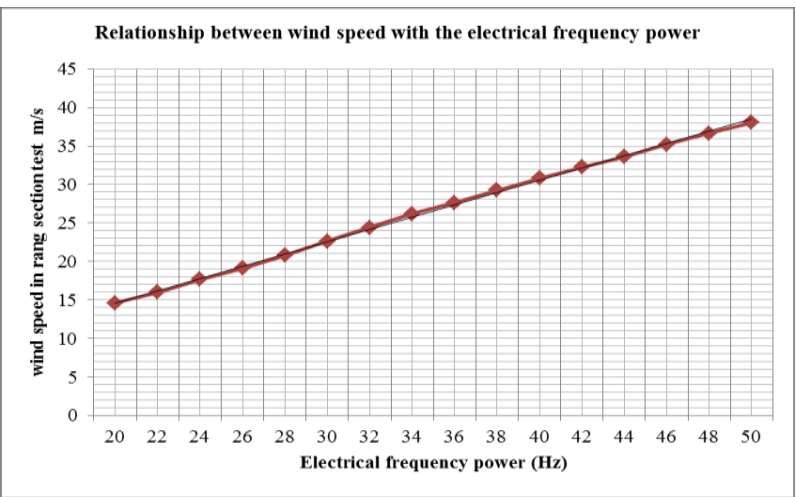

Fig 5. The relationship between wind speed values and frequency.

The wind speed values of the section test in Table 1. can be used to calculate the wind force pressure unit as follows

$$
q=0.004826 v^{2}
$$

where

$q$ is the wind force pressure unit $\left(\mathrm{km} / \mathrm{m}^{2}\right)$.

$v$ is the wind speeds $\mathrm{km} / \mathrm{hr}$.

From the experiment, asumming we select the maximum of the wind speeds of $38 \mathrm{~m} / \mathrm{s}$ or $136.8 \mathrm{~km} / \mathrm{hr}$ in Table 1 . we will obtain the wind force pressure is $90.31 \mathrm{~km} / \mathrm{m}^{2}$.

\section{Conclusion}

In this paper, a design and construction of a wind tunnel for engineering Laboratories are presented. Also, we conduct a sample experimental test of the opened loop wind tunnel with varity of frequency in the range of 20 $\mathrm{Hz}$ to $50 \mathrm{~Hz}$ increment by $2 \mathrm{~Hz}$. As the results, we obtain the wind speed values in the range of 14.50 meters per second to 38.50 meters per second. In addition, we found that the relationship between the wind speed values and freuqency is linear. This wind tunnel can be used for designing buildings with a maximum height 20 meters according to the Bangkok Regulations on Building Control 2001.

\section{References}

1. B. Jewel Barlow, H. William Rae, Jr. and A. Pope, Low speed wind tunnel Testing. New York : John Wiley, (1999)

2. C.F. Wilson, A.L. Camilletti, S.B. Calcutt and P.M. Ligrani. A wind tunnel for the calibration of Mars wind sensors. Planetary and Space Science.( 2008)

3. C. Huei Huang, C.Liang Lin and Jy Maw Chao. FERA in parameter identification with application in low-speed wind tunnel test. 15 : 495-509 Aerospace Science and Technology (2010)

4. R. Adrian Wittwer and V. Sergio Moller. "Characteristics of the low-speed wind tunnel of the UNNE. 84 : 307-320. Journal of Wind Engineering and Industrial Aerodynamics. (2000)

5. M. T. Boyle. "Low speed wind tunnel testing. 3139 Appears in Semiconductor Thermal and Temperature Measurement Symposium. SEMITHERM IV Fourth Annual IEEE. (2002) 